\section{(6) OPEN ACCESS}

\title{
Effectiveness of an injury prevention programme for adult male amateur soccer players: a cluster-randomised controlled trial
}

\author{
Anna M C van Beijsterveldt, ${ }^{1}$ Ingrid G L van de Port, ${ }^{1}$ Mark R Krist, ${ }^{1}$ \\ Sandor L Schmikli, ${ }^{1}$ Janine H Stubbe, ${ }^{2}$ Janet E Frederiks, ${ }^{3}$ Frank J G Backx ${ }^{1}$
}

${ }^{1}$ Department of Rehabilitation, Nursing Science and Sports, University Medical Centre Utrecht, Utrecht,

The Netherlands

${ }^{2}$ Netherlands Organization for Applied Scientific Research (TNO), Leiden, The Netherlands ${ }^{3}$ Royal Netherlands Football Association (KNVB) Sports Medical Centre, Zeist, The Netherlands

\section{Correspondence to} Anna-Marie van Beijsterveldt, Department of Rehabilitation, Nursing Science and Sports, University Medical Centre Utrecht, W01.121,

PO Box 85500, 3508 GA

Utrecht, The Netherlands; a.m.c.beijsterveldt@ umcutrecht.nl.

Received 14 April 2012 Accepted 10 July 2012

Published Online First 9 August 2012

\section{ABSTRACT \\ Background The incidence rate of soccer injuries is among the highest in sports, particularly for adult male soccer players. \\ Purpose To investigate the effect of the 'The 11 ' injury} prevention programme on injury incidence and injury severity in adult male amateur soccer players.

Study design Cluster-randomised controlled trial. Methods Teams from two high-level amateur soccer competitions were randomly assigned to an intervention ( $n=11$ teams, 223 players) or control group ( $n=12$ teams, 233 players). The intervention group was instructed to perform The11 in each practice session during one soccer season. The11 focuses on core stability, eccentric training of thigh muscles, proprioceptive training, dynamic stabilisation and plyometrics with straight leg alignment. All participants of the control group continued their practice sessions as usual.

Results In total, 427 injuries were recorded, affecting 274 of 456 players $(60.1 \%)$. Compliance with the intervention programme was good (team compliance $=73 \%$, player compliance $=71 \%$ ). Contrary to the hypothesis, injury incidences were almost equal between the two study groups: 9.6 per 1000 sports hours (8.4-11.0) for the intervention group and 9.7 (8.5-11.1) for the control group. No significant differences were found in injury severity, but a significant difference was observed in the location of the injuries: players in the intervention group sustained significantly less knee injuries.

Conclusions This study did not find significant differences in the overall injury incidence or injury severity between the intervention and control group of adult male soccer players. More research is recommended, focusing on injury aetiology and risk factors in adult male amateur soccer players.

\section{INTRODUCTION}

Participating in sports on a regular basis is considered a vital component of an active and healthy lifestyle to reduce the risk of various diseases and to contribute to better social and physical performance. To some extent, however, sports injuries are inevitable. The incidence rate of outdoor soccer injuries is among the highest of all sports, particularly for adult male soccer players. ${ }^{1} 2$ In the Netherlands, outdoor soccer causes the largest number of injuries each year (18\% of all sports injuries), totalling approximately 620000 injuries. ${ }^{3}$
Soccer is a high-intensity sport characterised by continuous changes of direction and high-load unipodal actions. Participating in soccer imposes high demands on neuromuscular control, agility and eccentric/concentric strength. Most soccer injuries are related to the lower extremities, in which muscle injuries are among the major problems. $^{4-6}$

Significant reductions of lower extremity injury risk have been reported to be achieved by intervention programmes focusing on intrinsic risk factors. $^{7-9}$ Eccentric strength training reduced the risk of hamstring injury in heterogeneous populations of soccer players. ${ }^{10-12}$ In addition, plyometric training and agility drills, the main components of a preventive programme developed by Heidt et $a l,{ }^{13}$ were found to be effective in lowering the incidence of injuries in soccer. It has also been shown that neuromuscular training can significantly reduce the risk of anterior cruciate ligament (ACL) injury in both male and female soccer players. ${ }^{14} 15$ Finally, balance training proved to be effective in reducing noncontact ACL injuries in soccer players, especially in female athletes. ${ }^{14}$

An exercise programme called 'The11', developed with the support of the World Football Association FIFA, also focuses on injury prevention. ${ }^{16}$ The effects of The11 on injury rates have been investigated previously. One study found that the programme significantly reduced injury rates (21\% fewer injuries) in male Swiss junior soccer players. ${ }^{17}$ However, this injury prevention effect was not observed in female Norwegian junior soccer players. ${ }^{18}$

The preventive effect of The11 has not been studied in male adult soccer players, who represent the largest group of active participants in soccer worldwide with high injury incidence rates. ${ }^{2} 19$ Therefore, our research on injury prevention focuses specifically on male adult soccer players As proposed by van Mechelen et al, ${ }^{20}$ our study addressed steps three and four of the prevention sequence model: introducing preventive activities to reduce future risk and/or severity of sports injuries, and assessing their effectiveness. The aim of the present study was therefore to investigate the effectiveness of The11 in this high-risk population of adult male amateur soccer players. We hypothesized that these exercises, when integrated in the warm-up of each practice session, would have a preventive effect on injury incidence and/or injury severity compared to usual practice sessions without The11. 


\section{METHODS}

\section{Trial design and randomisation}

In accordance with the principles of intention to treat, the effectiveness of the The11 injury prevention programme was evaluated in a two-armed cluster-randomised controlled trial. To minimise contamination, randomisation took place at cluster level, namely that of regional competitions. The trial was approved by the medical ethics committee of the University Medical Centre Utrecht and was registered in the Dutch trial register (NTR2416). For more detailed information regarding the methods, the reader is referred to the study protocol published elsewhere. ${ }^{21}$

\section{Participants}

Teams from two geographically separated districts in Dutch high-level amateur soccer were invited to participate in the study. Male players from these teams, who were aged between 18 and 40 years, were eligible for inclusion. They generally had two or three practice sessions and one match each week. Players who left the team during the season were included in the study, taking the time they spent on the team into account. All players provided a written informed consent at the start of the trial.

\section{Intervention}

The11 injury prevention programme has been developed with the support of the World Football Association FIFA. It includes 10 exercises focusing on core stability, eccentric training of thigh muscles, proprioceptive training, dynamic stabilisation and plyometrics with straight leg alignment. ${ }^{16}$ The 11 th component, fair play advice, was not included in the present trial. The11 included the following exercises: the bench, sideways bench, hamstrings, cross-country skiing, chest passing in single-leg stance, forward bend in single-leg stance, figures-of-eight in single-leg stance, jumps over a line, zigzag shuffle and bounding.

During the 2009-2010 soccer season (September-May), coaches of the teams in the intervention group were instructed to integrate The11 in the warm-up of each practice session (at least twice a week). Coaches were trained in applying The11 by the research staff at the end of the 2008-2009 season. In addition, coaches received a detailed information package (DVD, poster and reader) presenting the basic elements of the injury prevention programme. Coaches and players in the intervention group familiarised themselves with the programme during the 5 weeks preceding the start of the season (July-August), after which the programme was fully implemented in practice sessions at the start of the season. For players who are familiar with the exercises, the programme takes about 10-15 min. Coaches in the control group were invited to participate in a study of injury incidence and the characteristics of practice sessions. All participants in the control group continued their practice sessions as usual.

During the season, practice sessions of each team were visited by observers and members of the research staff each month. The purpose of these visits was to monitor the actual use of implementation of the The11 injury prevention programme in the intervention group. Random visits to the control group were scheduled to observe and record possible self-initiated preventive measures in their warm-up, specifically those of The11.

\section{Data collection procedure}

During the preseason, all players were asked to fill up a questionnaire to record baseline characteristics. During the 20092010 season, individual information about each participant's exposure to soccer (numbers of practice sessions and matches) was reported weekly by the coaches, using a computer-based recording form. When a player was not present at a regular practice session or game, the reason for his absence was reported on the exposure form as 'injured' or 'other'.

The team paramedic or sports trainer, who was present at every practice session and soccer match of the team, was responsible for recording the soccer injuries in both study groups. Therefore, he/she used the Web-Based Injury System (BIS) developed by the Netherlands Organization for Applied Scientific Research (TNO). ${ }^{21} 22$ BIS uses the basic guidelines of the consensus statement on injury definitions and data collection procedures in soccer. ${ }^{23}$ The system captures epidemiological information on sports injuries (location, duration and type), aetiology (intrinsic and extrinsic risk factors), consequences of injuries (eg, work/school/sports absenteeism) and the volume and type of medical treatment, using so-called injury and recovery forms.

\section{Outcomes}

Player characteristics recorded were age, height, weight, years of experience as a soccer player and soccer injuries sustained during the previous year (number and location).

The primary outcome of the study was the injury incidence per $1000 \mathrm{~h}$ of soccer participation (I). This was calculated according to the formula $\mathrm{I}=(\mathrm{n} / \mathrm{e}) \times 1000$, where $\mathrm{n}$ is the number of soccer injuries and e the total exposure time expressed as total hours of soccer participation. The Poisson model was used to obtain $95 \%$ confidence intervals (CI).

Exposure and all soccer injuries were recorded during the 2009-2010 competitive season, from the first competition match until the last regular competition match of the season. Table 1 shows the used definitions. These are in accordance with the consensus statement by Fuller et al. ${ }^{23}$ In addition, team and player compliance was recorded by all coaches in the intervention group using the exposure form.

Secondary outcomes were the absolute number of injuries, the proportion of injured players, as well as soccer injury characteristics (absenteeism, injury mechanism, recurrence, body part). Severity of injuries is reported as absenteeism in days. ${ }^{23}$

\section{Sample size}

Approximately $70 \%$ of all soccer players aged between 18 and 40 years (mainly men) get injured. ${ }^{24}$ Based on the results reported by Junge et $a l^{17}$ and Heidt et al, ${ }^{13}$ we estimated that the The11 programme would result in a $25 \%$ reduction of soccer injuries in our study. With a power of 0.80 and $\alpha$ of 0.05 , this meant that 90 players in each group had to take part in the study during an entire soccer season. Given an estimated

Table 1 Used definitions in data collection ${ }^{23}$

\begin{tabular}{ll}
\hline Injury & $\begin{array}{l}\text { Any physical complaint sustained by a player that results from a } \\
\text { soccer match or soccer practice session, irrespective of the need } \\
\text { for medical attention or time loss from soccer activities }\end{array}$ \\
Recurrent injury & $\begin{array}{l}\text { An injury of the same type and at the same site as a previous } \\
\text { injury and which occurs after a player's return to full participation } \\
\text { from the index injury }\end{array}$ \\
$\begin{array}{l}\text { Match } \\
\text { exposure }\end{array}$ & $\begin{array}{l}\text { Play between teams from different clubs } \\
\text { Training } \\
\text { exposure }\end{array}$ \\
& $\begin{array}{l}\text { Team based and individual physical activities under the control or } \\
\text { guidance of the team's coaching or fitness staff that are aimed at } \\
\text { maintaining or improving players' football skills or physical } \\
\text { condition }\end{array}$
\end{tabular}


inflation factor for cluster randomisation effects of $1.8,{ }^{18}$ and assuming a drop-out rate of $26 \%,{ }^{17}$ the research staff aimed to include a minimum of 219 players in each group at the start of the season. Assuming 19 players per team, 12 teams were included in each group.

\section{Statistical methods}

The statistical procedures were performed with SPSS 17 (SPSS Inc., Chicago, Illinois, USA) and R (V2.13.2). Baseline characteristics, measured as continuous variables, were expressed as mean and standard deviation (SD). Ordinal or categorical variables such as injury history were expressed as percentages. The following outcome parameters were analysed: injury incidence, proportion of injured players and injury profile. Because of their skewed distribution, exposure and absenteeism were presented as median and interquartile range (IOR). The categorical parameters representing injury profile were expressed as percentages.

The outcome parameters of the intervention and control groups were compared using a univariate T-test and MannWhitney U-test for the continuous parameters, and $\chi^{2}$ analysis for categorical parameters. Significant differences between the two study groups at baseline were included as covariates (ANCOVA) to test the intervention effect.

To evaluate any effect of the programme during the season, survival curves (based on Cox regression) for both study groups were compared. ${ }^{25}$ Additionally, Cox regression for recurrent events was used to compare the two groups, enabling both first time and recurrent injuries (adjusted for the time periods during each player had been on the team) to be used in the analysis. $^{26}$ Two-tailed $p$ values less than 0.05 were considered significant.

\section{RESULTS}

The initial study population consisted of 24 soccer teams; one team declined to participate. The two clusters with 23 teams were randomised, resulting in 11 teams in the intervention group and 12 teams in the control group. Shortly after randomisation, the coach of one team from the intervention group refused to use The11 during the practice sessions. Data of 456 players were analysed, 223 in the intervention group and 233 in the control group (figure 1). During the intervention season, 29 players $(6.4 \%)$ were lost to follow-up, mainly because they ended their soccer career or because they changed the team or club. All their available data were included in the analysis of the effects of the intervention programme. No significant difference in dropout rate was found between the intervention group $(n=11,4.9 \%)$ and the control group $(n=18,7.7 \%)$. Baseline characteristics of the players in the two study groups were similar, except for height and weight (table 2). Baseline data from dropouts and players with complete follow-up were not significantly different.

\section{Exposure and injury characteristics}

During the season, the players were involved in a total of $31518 \mathrm{~h}$ of practice time and $12734 \mathrm{~h}$ of match time, resulting in a total exposure time of $44252 \mathrm{~h}$. The mean practice and match times per player were 69.1 and $27.9 \mathrm{~h}$, respectively, during the 33 weeks of the competition season.

In all, 427 injuries were recorded, affecting 274 of the 456 players $(60.1 \%)$. The most commonly injured body parts $(n=408)$ were ankle $(19.1 \%)$, posterior upper leg $(15.9 \%)$, knee $(15.7 \%)$, anterior upper leg $(10.5 \%)$ and groin $(10.5 \%)$. The overall injury incidence for both groups was 9.6 (8.8-10.6)

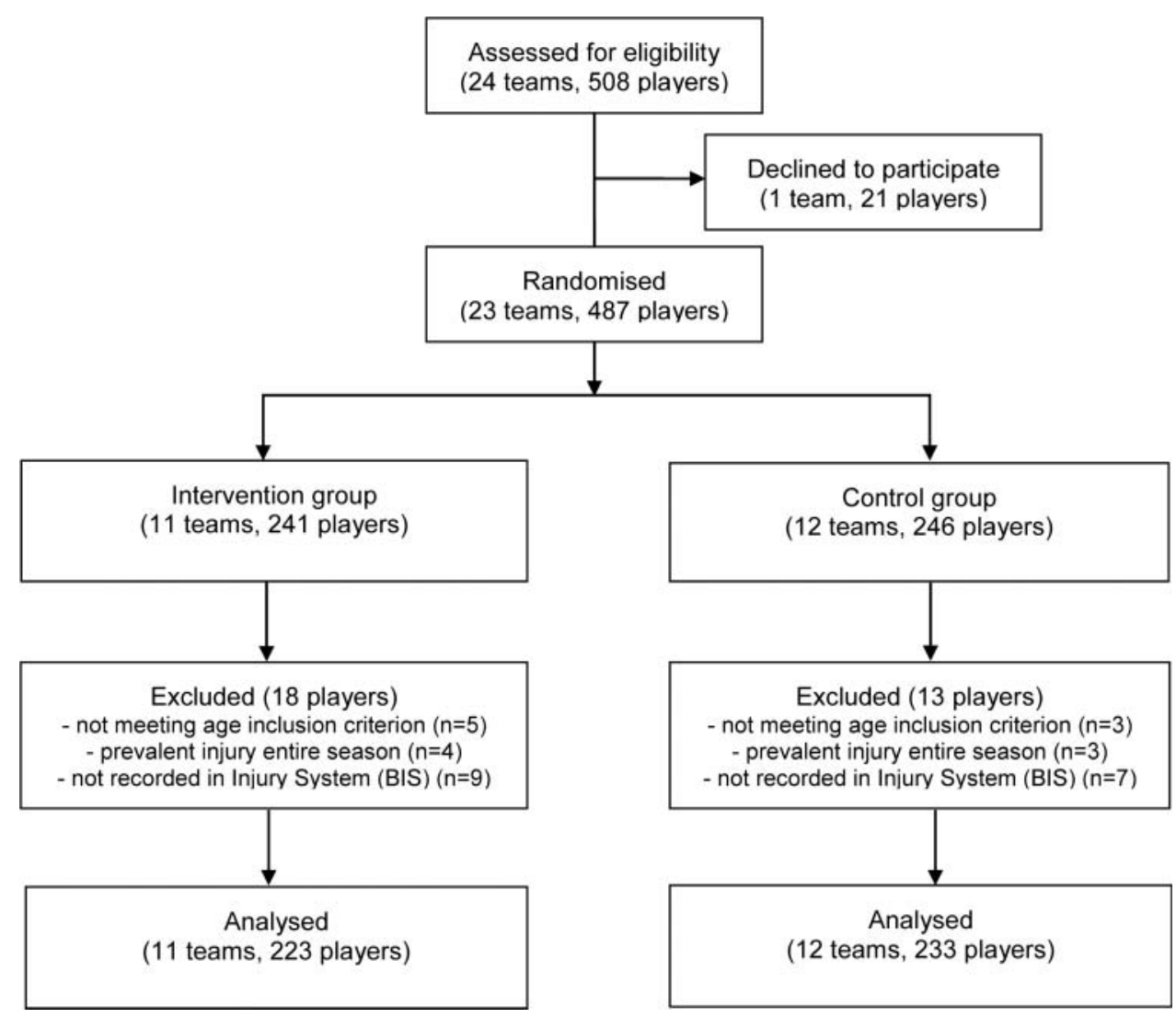

Figure 1 Flow diagram of the study population. 
Table 2 Baseline characteristics of the soccer players $(n=456)$

\begin{tabular}{lll}
\hline & $\begin{array}{l}\text { Intervention group } \\
\text { (mean } \pm \text { SD) }\end{array}$ & $\begin{array}{l}\text { Control group } \\
\text { (mean } \pm \text { SD) }\end{array}$ \\
\hline Age (years) & $24.4 \pm 4.1$ & $25.1 \pm 4.3$ \\
Height $(\mathrm{m})^{*}$ & $1.85 \pm 0.1$ & $1.82 \pm 0.1$ \\
Weight $(\mathrm{kg})^{*}$ & $79.1 \pm 7.4$ & $77.4 \pm 7.4$ \\
BMI $\left(\mathrm{kg} / \mathrm{m}^{2}\right)$ & $23.2 \pm 1.8$ & $23.3 \pm 1.8$ \\
Soccer experience (years) & $17.2 \pm 4.3$ & $17.7 \pm 4.6$ \\
$\quad$ Injury history (\%) & & \\
$\quad$ Injured in previous year & $73.4(\mathrm{n}=214)$ & $64.7(\mathrm{n}=221)$ \\
$\quad$ Injured at start of season & $11.7(\mathrm{n}=223)$ & $11.6(\mathrm{n}=233)$ \\
\hline
\end{tabular}

${ }^{*}$ Significantly different between the intervention and control group.

injuries per 1000 player hours; 3.4 (2.8-4.1) in practice sessions and 21.9 (19.5-24.6) in matches.

\section{Compliance}

Teams in the intervention group completed the intervention programme in $73 \%$ of all practice sessions (median 47, range 0-63), corresponding to performing The11 an average of 1.3 times per week. Players completed the exercises in $71 \%$ of the practice sessions they attended. Player absence meant that The11 was performed an average of 31 times per season (median 35, range 0-63). None of the teams in the control group regularly performed a structured prevention programme comparable to the intervention programme.

\section{Effects of the intervention programme}

As table 3 shows, overall injury incidences were almost equal for both groups: 9.6 per 1000 sports hours (8.4-11.0) for the

Table 3 Comparison of the intervention and control group

\begin{tabular}{|c|c|c|}
\hline & Intervention group & Control group \\
\hline Injuries & 207 & 220 \\
\hline Injured players (\%) & 60.5 & 59.7 \\
\hline Injury occurrence: match/training (\%) & $65.4 / 34.6(n=191)$ & $69.6 / 30.4(n=194)$ \\
\hline Hours of exposure (median, IOR) & $103.4,31.4$ & $104.3,35.0$ \\
\hline Total injury incidence (95\% Cl) & $9.6(8.4$ to 11.0$)$ & 9.7 (8.5 to 11.1$)$ \\
\hline Match injury incidence (95\% CI) & 21.1 (17.8 to 25.0$)$ & 22.7 (19.3 to 26.7$)$ \\
\hline $\begin{array}{l}\text { Practice injury incidence } \\
(95 \% \mathrm{Cl})\end{array}$ & 3.7 (2.8 to 4.8$)$ & $3.1(2.3$ to 4.0$)$ \\
\hline $\begin{array}{l}\text { Days of sports absenteeism } \\
\text { (median, IQR) }\end{array}$ & $14,28.5(n=204)$ & $17,30(n=211)$ \\
\hline Injury severity (\%): & $(n=205)$ & $(n=214)$ \\
\hline Slight ( 0 days) & 0 & 0.5 \\
\hline Minimal (1-3 days) & 5.9 & 5.1 \\
\hline Mild (4-7 days) & 18.5 & 21.5 \\
\hline Moderate (8-28 days) & 46.3 & 41.6 \\
\hline Severe ( $>28$ days) & 28.8 & 29.9 \\
\hline Career ending & 0.5 & 1.4 \\
\hline Injury mechanism: acute/overuse (\%) & $78.9 / 21.1(n=199)$ & $82.7 / 17.3(n=197)$ \\
\hline Recurrent injury (\%) & $13.0(n=193)$ & $14.1(n=193)$ \\
\hline Injury location (\%) (top 5) & $\begin{array}{l}(\mathrm{n}=206) \\
\text { 1. Ankle: } 21.8 \\
\text { 2. Upper leg } \\
\quad \text { (posterior): } 18.4 \\
\text { 3. Knee: } 11.7^{*} \\
\text { 4. Groin: } 9.7 \\
\text { 5. Upper leg } \\
\text { (anterior): } 8.3 \\
\text { 6. Other: } 30.1\end{array}$ & $\begin{array}{l}(\mathrm{n}=202) \\
\text { 1. Knee: } 19.8^{*} \\
\text { 2. Ankle: } 16.3 \\
\text { 3. Upper leg } \\
\quad \text { (posterior): } 13.4 \\
\text { 4. Upper leg } \\
\text { (anterior): } 12.9 \\
\text { 5. Groin: } 11.4 \\
\text { 6. Other: } 26.2\end{array}$ \\
\hline
\end{tabular}

$\mathrm{IQR}$, interquartile range; $\mathrm{Cl}$, confidence interval.

*Significantly different between the intervention and control group. intervention group and 9.7 (8.5-11.1) for the control group, as well as incidences of match and practice injuries. Nor were significant differences found in injury severity. None of the other outcomes showed significant differences between the two groups, apart from the percentage of knee injuries. However, after a Sidak correction for multiple testing $(n=6)$ this result should be interpreted with care. In addition, when exposure is taken into account, the difference in knee injuries was no longer significant. Results were corrected for baseline group differences in height and weight (table 3).

Cox regression was used to further analyse the effects of the intervention programme. Survival curves for the injuries (without re-injuries) throughout the season showed no significant difference between the two groups (figure 2). The analysis including all injuries that occurred during the season (ie, both first-time and recurrent injuries) yielded the same result.

\section{DISCUSSION}

In contrast to our hypothesis, we found no preventive effect of The11 on injury incidence or injury severity among male adult amateur soccer players during one season.

Whereas we found no preventive effect of The11 in our study population, consisting of adult men playing at a high amateur level, Junge et $a l^{17}$ found preventive effects of the programme among male youth soccer players. It is conceivable that an exercise programme have greater physical effects in younger players, since they have not yet established their basic movement patterns. ${ }^{27}$ This may explain why an effect was found in junior soccer players, but not in senior soccer players playing at the highest amateur levels.

A gender effect has been suggested, as The11 includes five exercises with a major focus on balance and knee alignment. ${ }^{28}$ These exercises aim to improve core stability and neuromuscular control, ${ }^{1529}$ and it is well known that female players have a 2-3 times higher ACL injury risk than male players, related to impaired knee alignment and lack of muscular balance. ${ }^{30} 31$ Hence, a preventive effect may be more likely in female soccer players than in male soccer players. ${ }^{13} 32$ The findings of the

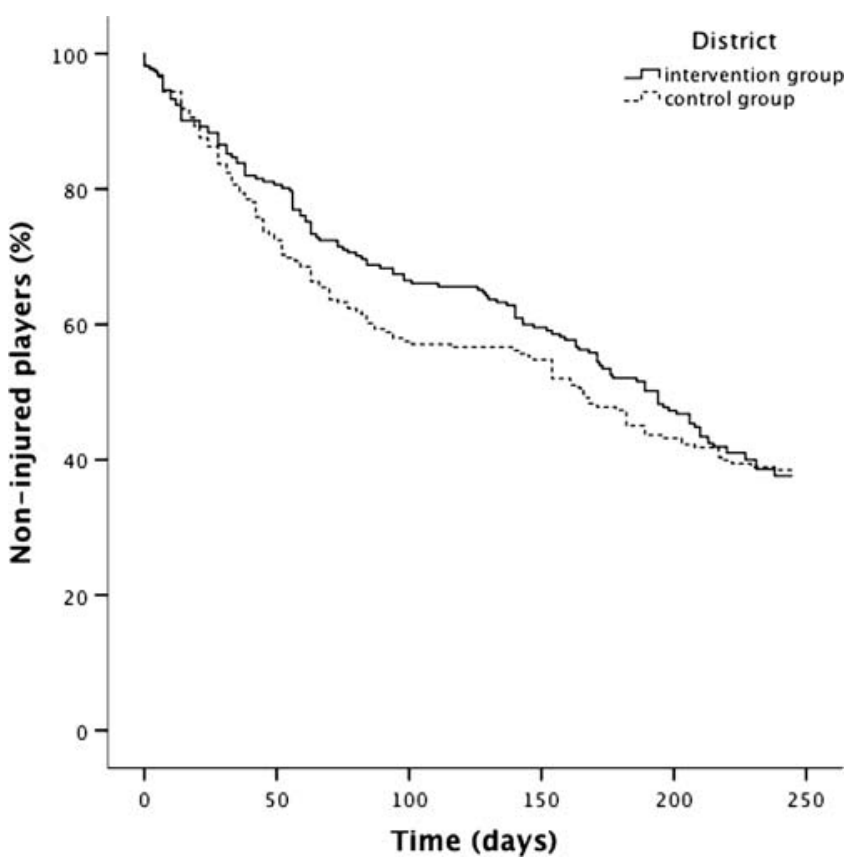

Figure 2 Survival curves based on Cox regression for first soccer injuries during the 2009-2010 season. 
present study among a male soccer population supports this. It is thus important that prevention programmes such as The 11 sufficiently address the specific injury risk factors relating to gender, age and playing level.

A study by Steffen et $a l^{18}$, which included female youth soccer players, reported no effects of The11. They suggested that low compliance with the programme explained the lack of effects and this was one of the most important reasons to introduce a modified programme: The $11+$. The $11+$ includes a greater diversity of exercises, changing both the type and the intensity during the soccer season. Subsequently, Soligard et al reported a preventive effect of The11+ among female youth soccer players. Among others, they reported a reduction in the incidence of knee injuries, which is to a certain extent in line with our results. ${ }^{27} 33$ The success of the new programme was partially explained by increased compliance to very acceptable levels $(77 \%)$. However, it seems unlikely that low compliance was a key factor in the lack of an effect achieved with the original The11 programme in our study, as compliance was almost as good as that reported by Soligard et al.

The positive effect found by Soligard et al could also suggest that the intensity of The11 may not have been sufficient to achieve adequate preventive effects in our study population. ${ }^{27} 33$ For example, exercise 3 of The11 is the so-called 'Nordic Hamstring' exercise. It has been shown in male soccer players that a gradual increase in the number of repetitions over 4 weeks-from two sets of 5 to three sets of 8-12 repetitionsincreases eccentric hamstring muscle strength and decreases the rate of hamstring strain injuries. ${ }^{10} 1234$ This graded protocol comes close to the one implemented in The11+. In contrast, the Nordic Hamstring exercise protocol in The11 contains only a single set of five repetitions, which does not vary through the season. This might suggest that the intensity of at least some of the exercises in The11 were not sufficient to decrease the injury rate in our adult male amateur soccer players.

Having found no positive effect of the prevention programme among male adult amateur soccer players, we need to return to step two of the model by van Mechelen. ${ }^{20}$ This means at least that a better understanding is needed of the aetiological factors and injury mechanisms as risk factors for soccer injuries in male adult soccer players.

This study was the first randomised controlled trial documenting the effects of The11 on male adult soccer players. Ideally, randomisation in trials should take place at the level of the subjects (players). Given the settings and methods in this trial, both practical and theoretical reasons made it impossible for such a randomisation to be applied. Alternatively, randomisation at the level of teams would have been the preferred route. However, it would not have been acceptable if some teams could possibly profit from the programme, while others in the same competition could not. As a result, randomisation had to take place at district level, which led to a higher number of players being included in the trial.

In view of the expected large number of injuries in this study, verification of the injury diagnosis by an independent medical doctor was impossible to implement. However, the recording of injuries as well as the diagnosis was assumed to be very reliable. Using the definitions in the consensus statement on injury definitions and data collection in soccer, ${ }^{23}$ injuries were recorded primarily by local, well-trained paramedics. Any injury that may have been missed was likely to be recorded in the weekly exposure form by the coaches. In case of any inconsistencies between the two recordings about the absence of a player due to injury, a member of the research staff contacted the coach and/or paramedic to verify the absence. Given these procedures, reporting bias and underreporting should have been minimal. If any under-reporting exists, it will be restricted to minor injuries because more than $70 \%$ of the injuries reported resulted in absence of more than 1 week.

In conclusion, there are serious doubts that a general, multicomponent training programme such as The11 is effective in this particular population of adult male amateur soccer players. The nonspecific content of the programme, an ineffective intensity, and possibly also the limited number of two training sessions per week available to perform the programme may have caused the programme to become ineffective. Unfortunately, the programme was tested as a single intervention, making it impossible to determine which exercises failed to have an impact on the injury risk. New research should focus on the correct type and dose-response relationship of exercises, specifically addressing risk factors for injuries in adult male amateur soccer players. Such research should at least cover the most frequently reported injuries being ankle, knee, upper leg and groin injuries.

\section{What are the new findings?}

Although in some studies The11 was used to reduce injury rates in various populations, its effectiveness has not yet been studied in the largest group of active participants in soccer worldwide: male adult soccer players. In our study among adult male amateur soccer players, The11 did not significantly reduce injury incidence or injury severity.

Correction notice This paper has been corrected since it was published Online First. The first author's first name has been corrected to Anna. Also in table three, in the row named 'Injury severity' the numbers in the Intervention and Control groups were wrong and these have been corrected.

Acknowledgements We are grateful to the district managements of the Royal Netherlands Football Association (KNVB), representatives of the participating soccer clubs (board members, coaches, and medical staff) and the soccer players involved.

Contributors In accordance with the Vancouver Protocol, all seven authors have substantially contributed to conception and design, acquisition of data or analysis and interpretation of data; drafting the article or revising it critically for important intellectual content; and final approval of the version to be published.

Funding This study was funded by the Netherlands Organization for Health Research and Development (ZonMw), reference number 50-50110-96-554, and the Royal Netherlands Football Association (KNVB). The Open Access fee was funded by the Netherlands Organisation for Scientific Research (NWO).

Competing interests None.

Ethics approval The study protocol was approved by the Medical Ethics Committee of the University Medical Centre Utrecht, reference number 08/263.

Provenance and peer review Not commissioned; externally peer reviewed.

\section{REFERENCES}

1. Inklaar H. Soccer injuries. I: Incidence and severity. Sports Med 1994;18:55-73.

2. Schmikli SL, de Vries WR, Inklaar $\mathrm{H}$, et al. Injury prevention target groups in soccer: injury characteristics and incidence rates in male junior and senior players. J Sci Med Sport 2011:14:199-203.

3. Consumer Safety Institute. Factsheet Blessures door veldvoetbal (in Dutch) [Factsheet on outdoor soccer injuries]. Amsterdam: Consumer Safety Institute, 2011.

4. Ekstrand J, Hagglund M, Walden M. Epidemiology of muscle injuries in professional football (soccer). Am J Sports Med 2011;39:1226-32.

5. Ekstrand J, Hagglund M, Walden M. Injury incidence and injury patterns in professional football: the UEFA injury study. Br J Sports Med 2011;45:553-8.

6. Emery CA, Meeuwisse WH, Hartmann SE. Evaluation of risk factors for injury in adolescent soccer: implementation and validation of an injury surveillance system. Am J Sports Med 2005;33:1882-91. 
7. Aaltonen S, Karjalainen $\mathrm{H}$, Heinonen $\mathrm{A}$, et al. Prevention of sports injuries: systematic review of randomized controlled trials. Arch Intern Med 2007;167:1585-92.

8. Emery CA, Meeuwisse WH. The effectiveness of a neuromuscular prevention strategy to reduce injuries in youth soccer: a cluster-randomised controlled trial. Br J Sports Med 2010;44:555-62.

9. Junge A, Dvorak J. Soccer injuries: a review on incidence and prevention. Sports Med 2004;34:929-38.

10. Arnason A, Andersen TE, Holme l, et al. Prevention of hamstring strains in elite soccer: an intervention study. Scand J Med Sci Sports 2008;18:40-8.

11. Askling C, Karlsson J, Thorstensson A. Hamstring injury occurrence in elite socce players after preseason strength training with eccentric overload. Scand J Med Sci Sports 2003:13:244-50.

12. Petersen J, Thorborg K, Nielsen MB, et al. Preventive effect of eccentric training on acute hamstring injuries in men's soccer: a cluster-randomized controlled trial. Am J Sports Med 2011;39:2296-303.

13. Heidt RS Jr, Sweeterman LM, Carlonas RL, et al. Avoidance of soccer injuries with preseason conditioning. Am J Sports Med 2000;28:659-62.

14. Alentorn-Geli E, Myer GD, Silvers HJ, et al. Prevention of non-contact anterior cruciate ligament injuries in soccer players. Part 2: a review of prevention programmes aimed to modify risk factors and to reduce injury rates. Knee Surg Sports Traumatol Arthrosc 2009;17:859-79.

15. Grindstaff TL, Hammill RR, Tuzson AE, et al. Neuromuscular control training programmes and noncontact anterior cruciate ligament injury rates in female athletes: a numbers-needed-to-treat analysis. J Athl Train 2006;41:450-6.

16. F-MARC. Football medicine manual. Zurich, Switzerland: Fédération Internationale de Football Association (FIFA), 2005:81-93.

17. Junge A, Rosch D, Peterson $L$, et al. Prevention of soccer injuries: a prospective intervention study in youth amateur players. Am J Sports Med 2002;30:652-9.

18. Steffen K, Myklebust G, Olsen OE, et al. Preventing injuries in female youth football—a cluster-randomized controlled trial. Scand J Med Sci Sports 2008;18:605-14.

19. FIFA. FIFA Big count 2006. Zurich, Switzerland, 2007: Fédération Internationale de Football Association (FIFA).

20. van Mechelen W, Hlobil H, Kemper HC. Incidence, severity, aetiology and prevention of sports injuries. A review of concepts. Sports Med 1992;14:82-99.

21. van Beijsterveldt AM, Krist MR, Schmikli SL, et al. Effectiveness and cost-effectiveness of an injury prevention programmeme for adult male amateu soccer players: design of a cluster-randomised controlled trial. Inj Prev 2011;17:e2.
22. Stege JP, Stubbe JH, van Hespen ATH, et al. Injuries in professional Dutch football: a prospective cohort study. Br J Sports Med 2008;42:544.

23. Fuller CW, Ekstrand $\mathrm{J}$, Junge $\mathrm{A}$, et al. Consensus statement on injury definitions and data collection procedures in studies of football (soccer) injuries. Br J Sports Med 2006:40:193-201.

24. Schmikli SL, Backx FJ, Kemler HJ, et al. National survey on sports injuries in the Netherlands: target populations for sports injury prevention programmes. Clin J Sport Med 2009;19:101-6.

25. Bahr R, Holme I. Risk factors for sports injuries - a methodological approach. $\mathrm{Br}$ J Sports Med 2003:37:384-92.

26. Twisk JW, Smidt N, de Vente W. Applied analysis of recurrent events: a practical overview. J Epidemiol Community Health 2005;59:706-10.

27. Soligard T, Myklebust G, Steffen K, et al. Comprehensive warm-up programmeme to prevent injuries in young female footballers: cluster randomised controlled trial. BMJ 2008;337:a2469.

28. Benjaminse A, Gokeler A, Fleisig GS, et al. What is the true evidence for gender-related differences during plant and cut maneuvers? A systematic review. Knee Surg Sports Traumatol Arthrosc 2011;19:42-54.

29. Caraffa A, Cerulli G, Projetti M, et al. Prevention of anterior cruciate ligament injuries in soccer. A prospective controlled study of proprioceptive training. Knee Surg Sports Traumatol Arthrosc 1996:4:19-21.

30. Vescovi JD, VanHeest JL. Effects of an anterior cruciate ligament injury prevention programme on performance in adolescent female soccer players. Scand J Med Sci Sports 2010;20:394-402.

31. Walden M, Hagglund M, Werner J, et al. The epidemiology of anterior cruciate ligament injury in football (soccer): a review of the literature from a gender-related perspective. Knee Surg Sports Traumatol Arthrosc 2011;19:3-10.

32. Mandelbaum BR, Silvers HJ, Watanabe DS, et al. Effectiveness of a neuromuscular and proprioceptive training programme in preventing anterior cruciate ligament injuries in female athletes: 2-year follow-up. Am J Sports Med 2005;33:1003-10.

33. Soligard T, Nilstad A, Steffen K, et al. Compliance with a comprehensive warm-up programmeme to prevent injuries in youth football. Br J Sports Med 2010;44:787-93.

34. Mjolsnes R, Arnason A, Osthagen T, et al. A 10-week randomized trial comparing eccentric vs. concentric hamstring strength training in well-trained soccer players. Scand J Med Sci Sports 2004;14:311-17. 